\title{
Title: Decadal Prediction Skill in the GEOS-5 Forecast System
}

Authors: Ham, Y.-G., M. M. Rienecker, M. Suarez, Y. V. Vikhliaev, B. Zhao, J. Marshak, G. Vernieres, and S. D. Schubert

Submitted to: $A G U$

\begin{abstract}
A suite of decadal predictions has been conducted with the NASA Global Modeling and Assimilation Office's GEOS-5 Atmosphere-Ocean General Circulation Model (AOGCM). The hindcasts are initialized every December from 1959 to 2010 following the CMIP5 experimental protocol for decadal predictions. The initial conditions are from a multi-variate ensemble optimal interpolation ocean and sea-ice reanalysis, and from the atmospheric reanalysis (MERRA, the Modern-Era Retrospective Analysis for Research and Applications) generated using the GEOS-5 atmospheric model. The forecast skill of a threemember-ensemble mean is compared to that of an experiment without initialization but forced with observed $\mathrm{CO}_{2}$. The results show that initialization acts to increase the forecast skill of Northern Atlantic SST compared to the uninitialized runs, with the increase in skill maintained for almost a decade over the subtropical and mid-latitude Atlantic. The annualmean Atlantic Meridional Overturning Circulation (AMOC) index is predictable up to a 5year lead time, consistent with the predictable signal in upper ocean heat content over the Northern Atlantic. While the skill measured by Mean Squared Skill Score (MSSS) shows $50 \%$ improvement up to 10-year lead forecast over the subtropical and mid-latitude Atlantic, however, prediction skill is relatively low in the subpolar gyre, due in part to the fact that the spatial pattern of the dominant simulated decadal mode in upper ocean heat content over this region appears to be unrealistic. An analysis of the large-scale temperature budget shows that this is the result of a model bias, implying that realistic simulation of the climatological fields is crucial for skillful decadal forecasts.
\end{abstract}

